\title{
INVESTIGATION OF SYNCHRONIZED ACQUISITION OF ELECTROCARDIOGRAM AND PHONOCARDIOGRAM SIGNALS TOWARDS ELECTROMECHANICAL PROFILING OF THE HEART
}

\author{
DevanshiN. Damani ${ }^{1}$, Divaakar SivaBaalaSundaram ${ }^{2}$, Shivam Damani ${ }^{3}$, AnoushkaKapoor ${ }^{2}$, Adelaide \\ M.Arruda-Olson ${ }^{1}$ and Shivaram P. Arunachalam ${ }^{4}$ \\ 1 \\ Department of Cardiology, Mayo Clinic, Rochester, MN 55902 \\ ${ }^{2}$ Department of Biomedical Informatics and Computational Biology (BICB), University of Minnesota, \\ Rochester, MN 55904 \\ ${ }^{3}$ Department of Ocean Engineering, Indian Institute of Technology, Chennai, Tamil Nadu, 60036, India \\ ${ }^{4}$ Department of Medicine \& Radiology, Mayo Clinic, Rochester, MN 55902
}

Corresponding Author: Devanshi N. Damani

Email: PoigaiArunachalam.Shivaram@mayo.edu

https://doi.org/10.34107/BiomedSciInstrum.57.04305

\begin{abstract}
Cardiac diseases are the leading cause of death in the world. Electrocardiogram (ECG and Phonocardiogram (PCG signals play a significant role in the diagnosis of various cardiac diseases. Simultaneous acquisition of ECG and PCG signals can open new avenues of signal processing approaches for electromechanical profiling of the heart. However, there are no standard approaches to ensure high fidelity synchronous data acquisition to enable the development of such novel technologies. In this work, the authors report results on various data capture positions that could lead to standardization of simultaneous ECG and PCG data collection. Presence of lung sounds, variations in posture, depth and frequency of breathing can lead to differences in the ECG-PCG signals recorded. This necessitates a standard approach to record and interpret the data collected. The authors recorded ECG-PCG simultaneously in six healthy subjects using a digital stethoscope to understand the differences in signal quality in various recording positions (prone, supine, bending, semi recumbent, standing, left lateral and sitting with normal and deep breathing conditions. The collected digitized signals are processed offline for signal quality using custom MATLAB software for SNR. The results indicate minimal differences in signal quality across different recording positions. Validation of this technique with larger dataset is required. Future work will investigate changes in characteristic ECG and PCG features due to position and breathing patterns.
\end{abstract}

Keywords: electrocardiogram, ECG, phonocardiogram, PCG, heart sound, cardiac disease

\section{INTRODUCTION}

Globally, cardiovascular diseases are the leading cause of death and account for $17 \%$ of the health care expenditure in the US [1,2]. A variety of approaches, ranging from physical examination to investigations — both invasive and non-invasive, are currently being used for the diagnosis and 